\title{
Recent updates in transcatheter aortic valve implantation
}

\author{
Jeonghwan Cho', Ung $\mathrm{Kim}^{2}$ \\ ${ }^{I}$ Division of Cardiology, Daegu Veterans Hospital; ${ }^{2}$ Division of Cardiology, Department of Internal Medicine, \\ Yeungnam University Medical Center, Daegu, Korea
}

\begin{abstract}
Transcatheter aortic valve implantation (TAVI) has evolved from a challenging intervention to a standardized, simple, and streamlined procedure with over 350,000 procedures performed in over 70 countries. It is now a novel alternative to surgical aortic valve replacement in patients with intermediate surgical risk and its indications have been expanded to cohorts with bicuspid aortic valves, low surgical risk, and younger age and fewer comorbidities. Attention should be paid to further reducing remaining complications, such as paravalvular aortic regurgitation, conduction abnormalities, cardiac tamponade, and stroke. The aim of this review is to provide an overview on the rapidly changing field of TAVI treatment and to explore past achievements, current issues, and future perspectives of this treatment modality.
\end{abstract}

Keywords: Aortic valve stenosis; Transcatheter aortic valve implantation; Surgery; Indications

\section{INTRODUCTION}

Since the first in vivo transcatheter aortic valve implantation (TAVI) performed by Alain Cribier in January 2002 [1], TAVI has been widely accepted as a method of treatment for severe aortic valve stenosis in inoperable patients, and as a reasonable alternative to surgical aortic valve replacement (SAVR) in moderate- and high-risk groups [2]. TAVI has undergone remarkable advances in the last 15 years, with over 350,000 procedures performed in over 70 countries [3]. Simultaneously with the development of TAVI procedures and methods of transcatheter heart valve (THV) production, TAVI has been undergoing a simplification and standardization process [3]. The aim of this review is to investigate: 1) changes in the clinical and anatomical indications for TAVI; 2) trends in the

Received: April 18, 2018, Revised: May 8, 2018

Accepted: May 29, 2018

Corresponding Author: Ung Kim, Division of Cardiology, Department of Internal Medicine, Yeungnam University Medical Center, 170, Hyunchung-ro, Namgu, Daegu 42415, Korea

Tel: +82-53-620-3832, Fax: +82-53-654-8386

E-mail: woongwa@yu.ac.kr incidence of complications according to the advances and progress in TAVI; and 3) synopsis, current issues, and future directions in the rapidly changing field of TAVI.

\section{Changes in TAVI indications}

SAVR has improved symptoms and survival rates in most patients with severe aortic stenosis [2]. However, the 2005 Euro Heart Survey revealed that old age and severe heart failure were predictors of 1-year survival rate in severe aortic stenosis, surgery and survival rates were not correlated, and 1.3 of all patients with severe heart failure or those of old age could not undergo surgery [4]. In the midst of this clinical situation, TAVI emerged. The indications for TAVI have significantly changed in the last 15 years. TAVI is now indicated for inoperable patients and patients at low to high risk. The indications have been recently expanded to patients at moderate risk. In line with the results of a study that reported excellent TAVI outcomes [5] and a randomized comparative study on the procedure [3], the 2017 American Heart Association (AHA)/American College of Cardiology (ACC)/Society of Thoracic Surgery (STS) Guideline extended the indications for TAVI to class I

Copyright (C) 2018 Yeungnam University College of Medicine

This is an Open Access article distributed under the terms of the Creative Commons Attribution Non-Commercial License (http://creativecommons.org/licenses/by-nc/4.0/) which permits unrestricted non-commercial use, distribution, and reproduction in any medium, provided the original work is properly cited. 
(level of evidence A) for inoperable patients with high risk, and to class II (level of evidence A-B) for patients with moderate risk [2]. A recent European treatment guideline included the results of Placement of Aortic Transcatheter Valve (PARTNER) trial A (high risk group), PARTNER B (inoperable patient group), PARTNER 2 (moderate risk group), CoreValve (high risk group), and Nordic Aortic Valve Intervention (NOTION) (moderate/ low risk groups) trials, in addition to those of Surgical Replacement and Transcatheter Aortic Valve Implantation (SURTAVI) (moderate risk group) trial. Although the current guideline recommends SAVR for patients with severe aortic valve stenosis and for moderate and low risk groups, it recommends TAVI for patients deemed inoperable by the heart team (class I, level of evidence B). The guideline states that the decision between SAVR and TAVI must be made by the heart team for patients who are at increased risk during surgical treatment (moderate-risk patients and patients with STS score or EuroSCORE II $\geq 4 \%$ ) [6. TAVI is preferred for patients of old age for whom the femoral artery approach (class I, level of evidence B) may be used [6].

\section{TAVI indications for specific population}

Attention has been given to the expansion of TAVI indications for specific patient groups. Research on this topic is being actively conducted today.

\section{Degenerated surgical bioprosthesis}

Percutaneous valve-in-valve ( $\mathrm{ViV})$ insertion has been receiving much attention as a relatively safe and less invasive treatment from patients with failed surgical bioprosthetic valve insertion [7]. Based on data from registry clinical studies, selfexpandable CoreValve ${ }^{\circledR}$ (Medtronic, Minneapolis, MN, USA), balloon expandable SAPIEN XT ${ }^{\circledR}$, and SAPIEN $3^{\circledR}$ transcatheter heart valve (THV) (Edwards Lifesciences Corp., Irvine, CA, USA) have been approved for use in high-risk patients for whom surgical bioprosthetic valve insertion has failed. A multicenter registry study has reported that failure of surgical bioprosthetic valve due to stenosis and degeneration is associated with the use of a small valve size [7]. From the technical point of view, ViV TAVI reduces the incidence of paravalvular aortic regurgitation (PAR) and the frequency of new cardiac pacemaker insertions compared to natural aortic valve
TAVI. However, it increases the risk of coronary artery obstruction and the frequency of patient-prosthesis mismatch.

Two prospective studies using PARTNER $2 \mathrm{ViV}$ registry and CoreValve US Expanded Use have been recently published $[8,9]$. The former registered 96 patients in the early research period and added 265 patients under the same protocol following the primary research completion. The postoperative 30day mortality and 1-year mortality rates were $2.7 \%$ and $12.4 \%$ in the entire cohort. The 1-year mortality of the first 96 patients was 20\%, and that of follow-up patients was $9.8 \%$, showing a significant improvement in the mortality rates along with improvement in clinicians' skills [9]. The CoreValve US Expanded Use study included 223 patients with symptoms caused by impaired function of a surgically-treated valve. The allcause mortality was $2.2 \%$ at 30 days after surgery and $14.6 \%$ at 1 year. Moderate or severe PAR occurred in 3.5\% of patients. The predictors of high transvalvular pressure gradient at the time of discharge were the size of inserted valve at the time of surgery, the degree of stenosis of failed valve, and patientprosthesis mismatch [8-10]. Complications related to ViV TAVI can now be significantly prevented by accurate preoperative screening tests and through technical advance [10]. Important challenges of ViV TAVI treatment in patients with a small-sized surgical bioprosthetic valve remain higher postoperative transvalvular pressure gradient and late mortality rates than those of other patients with ViV TAVI. It was reported that positioning the THV slightly above the aortic annulus or in the annulus can reduce this phenomenon [10]. Researchers have proposed a technique of fracturing the inserted bioprosthetic valve ring using a high-pressure balloon as a way to make the process of ViV TAVI easier. This strategy reduced transvalvular gradients, increased useful cross-sectional area of the valve, and enabled additional THV expansion [11]. If the technique produces good results, TAVI may become the preferred method of treatment for patients with failed surgical bioprosthetic valve. The development of customized equipment for these patients is also anticipated.

\section{Bicuspid aortic valve}

Bicuspid aortic valve has high prevalence in relatively young patients with aortic valve stenosis, and accounts for $20 \%$ of all surgical treatments in elderly persons aged 80 years or older [12]. In the early period after development, TAVI produced 
unsatisfactory outcome in patients with bicuspid aortic valve due to the cusps that are more oval compared to those of the normal aorta, irregular, and severely calcified, as well as due to calcified raphe. In a study on first generation THVs, 15.9\% of patients with aortic stenosis and a bicuspid valve treated with TAVI showed moderate or severe PAR, and the incidence of THV malposition was also high [12]. Yoon et al. [13] recently published a study on clinical outcomes of patients who underwent TAVI for bicuspid and tricuspid valve stenosis. They reported a higher incidence of aortic root damage, second THV implantation, and PAR among patients with bicuspid valve stenosis. In their study, the incidence of complications caused by TAVI was the highest among patients with type 1 bicuspid valves and severely calcified raphes [13]. Based on the currently available findings, bicuspid aortic valve should not be excluded as an indication for TAVI. However, the outcomes may not be as excellent as that for tricuspid valve. Considering the variations in the aortic structure and the location of valvular calcium, the indications for TAVI must be carefully reviewed.

\section{Low-risk population}

Although limited data are available about the effect of TAVI on low-risk populations, publication of the results of PARTNER 3 and Low-Risk Evolut clinical trials is expected. The NOTION study is the only randomized trial on TAVI and SAVR in a low-risk population, in which $82 \%$ of 280 low-risk patients were randomly exposed to TAVI or SAVR in a 1:1 ratio [14]. The primary endpoints were combined cardiovascular events that occurred over 2 years, including all-cause death, cerebrovascular infarction, and myocardial infarction. No difference was found in these endpoints between the two groups ( $15.8 \%$ vs. $18.8 \%, p=0.43$ ) [14]. Similar to the results of a previous study on high-risk and moderate-risk populations, the ratio of cardiac pacemaker implantation and PAR was high among patients who underwent TAVI, and the risk of hemorrhage, cardiogenic shock, acute renal failure, and preoperative and postoperative atrial fibrillation was higher among patients who underwent SAVR [14]. By applying the experience of moderate-risk populations with TAVI to low-risk populations and detecting certain anatomical and technical factors that can choose between TAVI or SAVR in low-risk populations, it may become possible to choose the best method of treatment for these patient groups $[15,16]$.

\section{Aortic valve regurgitation}

The role of TAVI in the treatment of aortic valve regurgitation has been extremely insignificant, and it has been used for off-label indications in extremely high-risk populations. Currently approved TAVI instruments have been designed for the purpose of calcified aortic valve stenosis treatment. There is almost no data about the safety and efficacy of TAVI for the treatment of severe aortic valve regurgitation. JenaValve ${ }^{\mathrm{TM}}$ (JenaValve Technology, Munich, Germany) is the only commercially available THV that obtained the Conformité Européene mark for the treatment of patients with severe aortic valve regurgitation, inoperable patients, and high-risk patients, and uses the transapical approach [17]. Some studies have reported successful TAVI using THV and transfemoral approach [17]. Compared to TAVI in aortic valve stenosis, TAVI for pure aortic valve insufficiency is much more difficult to perform, and have a lower success rate, safety, and clinical efficacy. The risk of THV mobilization and migration is high in patients with pure aortic valve regurgitation, since they do not have a calcifiedaortic valve and aortic annulus. In fact, THV of the previous generation produced poor clinical outcomes due to THV embolization, migration, and PAR. However, Sawaya et al. reported that by using the new generation THV in patients with the same disease, the success rate for device installation could be increased up to $85 \%$ (54\% vs. $85 \%, p=0.011$ ) [18]. Preclinical and clinical studies are being conducted on a new THV (J-Valve ${ }^{\mathrm{TM}}$, JieCheng Medical Technology Co., Ltd., Suzhou, China) designed for installation on non-calcified aortic valves, and the indications for TAVI might be expanded to patients with aortic valve regurgitation [19].

\section{Procedural advances and complication trends}

\section{Trends of complication}

Despite the remarkable advances in THV production and implantation techniques, some serious complications should be still considered.

In the early period after TAVI introduction, vascular complications, neurologic events associated with the procedure, 
such as cerebral infarction, conduction abnormalities, and PAR were suggested as TAVI complications [3]. Attention on aortic rupture and coronary artery obstruction has recently been increasing as these complications, despite their low incidence, can be lethal [9]. Since the indications for TAVI have gradually been expanding to young and low-risk patients, long-term durability and intravalvular thrombosis have emerged as important issues to be considered [20-22].

\section{Procedure and THV advances}

New TAVI THVs allow accurate positioning of THV in the aortic annulus, have reduced delivery catheter size (14 Fr) to reduce related complications, and reinforced functions to enable intraoperative installation [23].

TAVI THVs that are currently approved for clinical use are SAPIEN $3^{\circledR}$ (Edwards Lifesciences Corp., Irvine, CA, USA), Evolut $\mathrm{R}^{\circledR}$ (Medtronic, Minneapolis, MN, USA), LOTUS ${ }^{\mathrm{TM}}$ (Boston Scientific, Marlborough, MA, USA), ACURATE neo ${ }^{\text {TM }}$ (Symetis, Ecublens, Switzerland), Portico ${ }^{\text {TM }}$ (St. Jude Medical, St. Paul, MN, USA), and Allegra ${ }^{\mathrm{TM}}$ (New Valve Technology, Hechingen, Germany). They can be categorized into SAPIEN $3^{\circledR} /$ Evolut $^{\circledR}{ }^{\circledR}$ Portico $^{\mathrm{TM}}$, ACURATE neo ${ }^{\mathrm{TM}}$, and Allegra ${ }^{\mathrm{TM}} /$ LOTUS $^{\mathrm{TM}}$ [24].

SAPIEN $3{ }^{\circledR}$ THV is designed as a cobalt-chrome structure with three leaflets inside a cobalt-chrome structure. Compared to SAPIEN XT ${ }^{\circledR}$ from the previous generation, SAPIEN $3^{\circledR}$ has a reduced diameter (14 Fr), and its valve cell structure was reinforced by introducing an open upper cell and a closed lower cell. Furthermore, an external seal was added to reduce PAR.

Evolut $\mathrm{R}^{\circledR}$ THV consists of a tricuspid heart valve sealed inside a self-expanding nitinol structure. It has been redesigned from the CoreValve devices from previous generations to gain an optimized radial force during valve implantation, and to enable retrieval and repositioning. Evolut PRO ${ }^{\circledR}$, which has been recently approved by the Food and Drug Administration (FDA), consists of the same platform as Evolut $\mathrm{R}^{\circledR}$, with additional external parts added to decrease the gap between the valve and the distal aorta, and to reduce PAR. Table 1 compares SAPIEN THV, which is the most commonly used THV, with CoreValve.

LOTUS $^{\mathrm{TM}}$ THV is a tricuspid valve supported by a sealed nitinol frame. It is the only THV that allows complete retrieval and repositioning even after the valve is completely implanted. Although it has the lowest incidence of PAR among all approved THVs (Fig. 1), it leads to conduction abnormalities requiring pacemaker insertion in $30 \%$ of patients following valve insertion (Fig. 1) [24]. The new generation LOTUS Edge $^{\mathrm{TM}}$ THV (Boston Scientific, Marlborough, MA, USA) with Depth Guard ${ }^{\mathrm{TM}}$ release mechanism (Boston Scientific, Marlborough, MA, USA) has been developed to lower the incidence of conduction abnormalities, and is still being tested in clinical trials with results waiting to be released.

Portico $^{\text {TM }}$ (St. Jude Medical, St. Paul, MN, USA) valve is

Table 1. Comparison between SAPIEN valve and CoreValve

\begin{tabular}{lll}
\hline \multicolumn{1}{c}{ Characteristic } & \multicolumn{1}{c}{ SAPIEN valve } & CoreValve \\
\hline Morphology & & \\
& & \\
Ptructure & Porcine pericardium & Bovine pericardium \\
Delivery & Balloon expandable & Self expandable \\
Vascular access & $18 \mathrm{~F}$ (XT) & 14 F (Evolut R) \\
Rapid pacing & Required & Not always \\
Left ventricular apical approach & Possible & Not possible \\
Retrievability & Not possible & Possible \\
Potential conduction abnormalities & Less & High \\
\hline
\end{tabular}




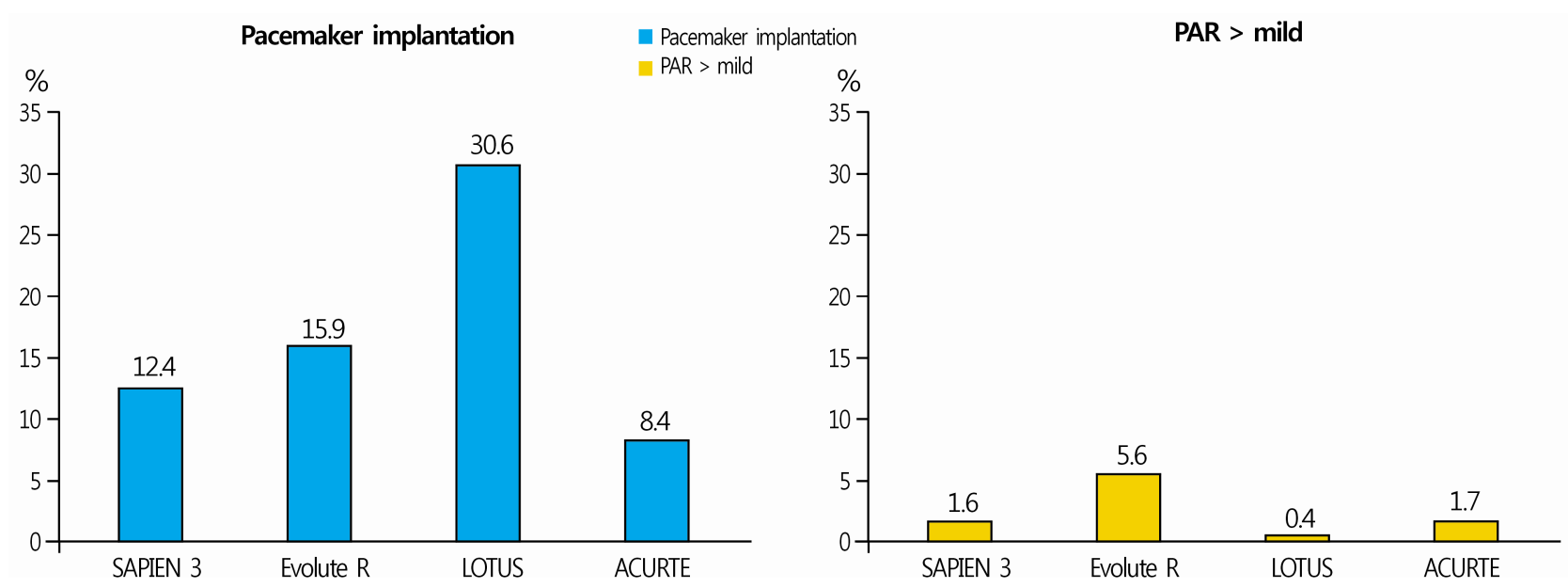

Fig. 1. Comparison of the incidence of permanent pacemaker implantation and more than mild PAR in patients with new-generation TAVI devices [21]. PAR, paravalvular aortic regurgitation; TAVI, transcatheter aortic valve implantation.

a self-expansive stent. The main characteristic of this THV is that it can be repositioned multiple times, and the coronary artery entrance can be easily found due to the large stent cells. ACURATE neo ${ }^{\mathrm{TM}}$ (Symetis, Ecublens, Switzerland) valve is a self-expanding nitinol stent, and consists of three stabilization arches for axial alignment on the aortic annulus, an upper fixture for positioning on the aortic annulus, and an open lower body. Although this THV cannot be retrieved or repositioned, it is extremely stable during implantation.

\section{Access routes and vascular complications}

Early TAVI procedures used the anterograde transseptal approach. Now, the transfemoral approach is the most preferred access route. Alternative routes (transapical, transaortic, and trans-subclavian) have additionally been developed, and their executability and reliability have been verified [25]; they must be assessed in a large number of patients. New assessment must consider clinician's level of experience, situations within individual hospitals, and patient's anatomical structures.

Historically, vascular complications have been important limitations of TAVI alongside the amount of blood loss, amount of blood transfusion required, and hemodynamic instability [26. However, with advances leading to miniaturization of delivery systems, improved patient selection and choice, and increased clinicians' experience, the incidence of lethal vascular complications has been reduced [26]. Today, vascular complications usually occur as minimal hemorrhage or vascular injury that can be managed through percutaneous surgery, such as arterial wall dissection and arterial obstruction [26].

\section{Cerebrovascular events}

Cerebrovascular events have shown high morbidity and mortality, and were a major topic of interest in the early period after TAVI was introduced [3]. In a large-scale meta-analysis including 72,318 patients from 64 studies, the 30-day incidence of cerebrovascular events was 3.3\% [27]. Many potential predictors of cerebrovascular events following TAVI have been identified, and most episodes of cerebral embolism that occur during TAVI were thought to occur during balloon angioplasty, manipulation of a catheter on the aortic valve, and valve implantation [3]. However, it has been revealed that about $50 \%$ of all cerebrovascular events caused by TAVI occur in the 24 hours following TAVI [28]. These cerebrovascular events are believed to originate from thrombus formation (e.g., newly developed atrial fibrillation), and indicate that the patient with TAVI might develop other diseases. Although cerebrovascular events present clear clinical symptoms, most patients treated with TAVI do not show clinical symptoms. However, it has been found that patients treated with TAVI who do not show clinical symptoms may show new cerebral ischemic lesions on brain magnetic resonance imaging [3]. According to the results of a recent study, the incidence of cerebrovascular events decreased by $2.5 \%$ over time compared to that of early TAVI procedures [23]. This trend can be explained by improved equipment systems and operator's level of experience owing to technical advances. 
In addition, several brain protection devices have been developed to prevent cerebrovascular events that occur during or immediately after TAVI [29], including Sentinel ${ }^{\mathrm{TM}}$ (Claret Medical, Inc., Santa Rosa, CA, USA) and TriGuard ${ }^{\mathrm{TM}}$ (Keystone Heart, Tampa, FL, USA). They were reported to reduce the size and number of subclinical/asymptomatic cerebral embolisms during TAVI [29]. Although Sentinel ${ }^{\mathrm{TM}}$ is the only FDAapproved device, it has failed to show meaningful reduction in cerebral infarction in clinical trials.

\section{Conduction abnormalities}

Since the aortic valve is located proximally to the cardiac conduction system, conduction abnormalities are frequently observed in patients treated with TAVI. Left bundle branch block (LBBB) is the most common conduction abnormality following TAVI, and complete atrioventricular block is the most important conduction abnormality that requires permanent cardiac pacemaker insertion. The frequency of cardiac pacemaker insertion following TAVI depends on the type of THV used. Conduction abnormalities can lead to left ventricular dyssynchrony, reduced left ventricular ejection fraction, increased hospitalization period, and increased need for cardiac pacemaker in the early and late period [30]. Preoperative anatomical evaluation of the valve, and selection of the most appropriate device are crucial for reducing the incidence of conduction abnormalities [30]. However, the problem of conduction abnormalities could not be completely resolved even after the development of second generation THV (Evolut $\mathrm{R}^{\circledR}$, LOTUS $^{\mathrm{TM}}$ ) and addition of the possibility of THV retrieval and repositioning [24].

\section{Paravalvular aortic regurgitation (PAR)}

PAR is a complication of prosthetic aortic valve replacement that occurs more frequently following SAVAR than after TAVI [3,31]. The reported incidence of moderate or severe grade PAR following TAVI using a first generation THV was $12-21 \%$ [31]. PAR of moderate grade or above has clinical associations, and increases the risk of all-cause mortality [32]. The three major causes of PAR that occur during TAVI are severe valvular calcification, setting a small THV size before the procedure, and unstable THV binding within the aortic circumference due to THV displacement. A drastic decrease in the incidence of severe PAR has been observed over time [24]. This fact is related to the possibility to measure the aortic annular size before surgery owing to the advances in medical imaging, more wide distribution of next generation THVs with higher predictability due to the valve repositioning function, and the development of structures with an external suture tape that can fill the space between THV and aortic wall The incidence of PAR, although reduced, varies among different THV systems (Fig. 1).

\section{Coronary occlusion and aortic root rupture}

Based on current TAVI data, the incidence of coronary artery obstruction and aortic root or aortic annulus rupture is generally below $1 \%[10]$. The obstruction of coronary arteries in the natural aortic valve occurs because of a calcified valve of large size or the external suture leaflet on the opening of coronary artery being displaced due to TAVI. Narrow Valsalva, severe cusp calcification, and low positioning of coronary arteries opening are major predictors of coronary obstruction during TAVI [10]. When performing aortic angiography and balloon angioplasty before surgery, it is necessary to check if the coronary artery opening is getting obstructed as the balloon expands. When the balloon is completely expanded, the coronary opening bumps into the Valsalva wall due to the calcified cusp, and may become obstructed [33]. A common strategy when the risk of coronary obstruction is high due to TAVI is to insert a wire and balloon or stent in advance, and then perform TAVI. If coronary obstruction occurs, an emergency procedure is performed.

Aortic root rupture has poor acute prognosis [10]. Two important characteristics, moderate to severe LVOT/subannular calcification and THV oversizing, in which the produced valve is larger than the aortic annulus, are associated with aortic root rupture and para-aortic hematoma. These complications can be prevented by carefully choosing the THV type and size.

\section{Transcatheter heart valve failure}

TAVI is currently performed in relatively young patients with low risk. The average life expectancy is gradually being used for relatively young patients and low-risk patients, and is expected to exceed that of patients who underwent early TAVI procedures, which is related to long-term durability 
of THV. Failure of THV implantation can present as stenosis (calcification, pannus, or thrombosis) or artificial valve regurgitation (reduced cusp mobility, cusp ripping, or endocarditis) [20]. In PARTER I study that monitored patients for 5 years, no significant increase in the mean THV gradient or structural valvular worsening was observed [20]. Three studies have reported on the 5-year progress following treatment with SAPIEN or CoreValve [20]. In two of these studies, no problem related to the durability of THV and no significant change in transprosthetic gradient were detected. The incidence of THV dysfunction was $3.4 \%$ and $4.2 \%$ in each study [20]. Although thrombosis rarely occurs as a result of failed THV implantation, it has drawn clinical attention [21,22]. With a recent observation of normal transannular pressure gradient in association with subclinical leaflet thrombosis, reduced cusp mobility has been reported to affect both the percutaneously-inserted cardiac valve and the surgically treated valve [22]. In RESOLVE and SAVORY studies, subclinical leaflet thrombosis could be diagnosed by computed tomography (CT) in $12 \%$ of 890 patients. Five of 138 patients (3.6\%) with surgically implanted valves and 101 of 752 patients treated with TAVI (13.4\%) showed subclinical leaflet thrombosis [22]. Although no difference was found in the incidence of stroke between patients with reduced valve mobility and those without, the risk of transient ischemic attack was significantly higher in patients with subclinical leaflet thrombosis diagnosed by CT, than in those without (6\% vs. $1 \% ; p<0.001$ ) [22]. These data show that although dual antiplatelet therapy (DAPT) has been the standard method of treatment since TAVI, anticoagulation therapy may be useful in the future. The incidence of reduced cusp mobility following TAVI was significantly lower in patients who received DAPT ( $3.6 \%$ vs. $14.9 \%, p<0.001$ ) than in those who received oral anticoagulants, and non-vitamin $\mathrm{K}$ oral anticoagulants prevented thrombosis as effectively as warfarin [22]. A global study compared the rivaroxaban antithrombotic strategy to an antiplatelet-based strategy after transcatheter aortic valve replacement to optimize clinical outcomes (GALILEO), while another trial assessed the anti-thrombotic strategy after trans-aortic valve implantation for aortic stenosis (ATLANTIS). Currently, clinical trials are being conducted in which a non-vitamin $\mathrm{K}$ oral anti-coagulant (Rivaroxaban ${ }^{\circledR}$, Apixaban ${ }^{\circledR}$ ) is administered to prevent valve thrombosis and other symptoms in patients who underwent TAVI. The current 2017 ACC/AHA guideline recommends the use of war- farin for 3 months following TAVI [2].

Although the incidence of infective endocarditis is low (0.53.1\%) after TAVI, infective endocarditis is one of the most serious complications of TAVI [33]. In a multicenter registry study on 250 cases of postoperative infective endocarditis, the in-hospital mortality rate was 36\%, and the 2-year mortality rate was $66.7 \%$ [34]. Young age, male sex, diabetes mellitus, and moderate to severe residual aortic regurgitation were associated with increased risk of infective endocarditis [34].

Although THV failure and organ durability are major topics of interest, TAVI reoperation has been found to be safe and effective based on mortality and morbidity in comparison with redo valve surgery [35].

\section{Procedure optimization}

One of the biggest issues surrounding TAVI is the standardization and simplification of TAVI procedure. Many researchers have endeavored to create a program aimed at the preoperative, intraoperative, and postoperative standardization and simplification of TAVI in the last few years [36-38].

\section{Preoperative optimization}

TAVI requires several preliminary assessments and tests for confirming clinical and anatomical indications by echocardiography and CT. These tests are generally to be performed without hospitalization. The TAVI team must think beyond the traditional clinical and anatomical criteria of TAVI indications, but consider the clinical, nonclinical, and psychological factors that can affect postoperative recovery. These factors include the patient's will to participate in heart rehabilitative therapy, likelihood of getting Alzheimer's disease, and family support.

\section{Intraoperative optimization}

For the stabilization of TAVI, optimization and simplification of the procedure are necessary (Table 2). Although many operators perform TAVI in a hybrid operating room, TAVI can be safely performed in a common cardiac catheterization room. If the femoral approach is being used, the presence of a cardiac surgeon is not required. However, it is important that a cardiac surgeon participates in the evaluation process and manage potentially lethal complications. Performing TAVI 
Jeonghwan Cho et al.

Table 2. Technical considerations during TAVI procedures

\begin{tabular}{ll}
\hline Specific procedures during TAVI & Situations to be considered \\
\hline General anesthesia & Hemodynamically unstable \\
& TEE guidance \\
TEE assistance & Very nervous to mild sedation \\
Surgical assistance for femoral approach & High risk of paravalvular leakage \\
Balloon pre-dilatation & Valve in valve TAVI for stentless surgical valves \\
& Heavy obesity \\
& Severe calcification on femoral arteries \\
& Bicuspid aortic valves \\
\hline
\end{tabular}

TAVI, transcatheter aortic valve implantation; TEE, transesophageal echocardiography.

via the femoral approach under local anesthesia, and not performing back-up transesophageal echocardiography and balloon angioplasty are one of the strategies to reduce the invasiveness and cost of the procedure. In addition, a temporary pacemaker must be removed at the end of the procedure if there is no concern about conduction abnormalities.

\section{Postoperative optimization}

Following the procedure, special attention must be given to patient's hemodynamics and heart rate. They must be carefully monitored in the coronary intensive care unit or operating room for at least 10-15 minutes. Next, the patient's clinical conditions must be thoroughly assessed according to the procedure outcome, and electrocardiography and echocardiography data. If there is no hematoma near the blood vessels and no bleeding problem, and the pacemaker has been temporarily removed, the patient must be allowed to move after a few hours, and must be discharged as soon as possible if he/she has no complications.

Early discharge (within 24-72 hr) after TAVI has been found to improve patients' motor function and accelerate recovery without compromising the safety of the procedure; this approach has also been confirmed by the experience on patients in Europe and North America [34,35]. Based on clinical experience, conduction abnormalities, hemorrhage, and acute renal injury are the factors that most frequently extend the hospitalization period. Consistent monitoring of acute A-V block is necessary. TAVI has been actively performed in many domestic institutions. However, the procedure places a huge financial burden on patients due to its cost. TAVI may be perfor- med even more actively if the procedure is simplified to reduce the cost and health insurance coverage is provided for TAVI.

\section{CONCLUSION}

Although TAVI is a complex procedure, significant advances have been made through the simplification and standardization of the procedure, while other improvements are still underway. It is anticipated that through consistent technical advances and simplification of the procedure, TAVI will be more widely used, and be accepted as a safe procedure.

\section{CONFLICT OF INTEREST}

No potential conflict of interest relevant to this article were reported.

\section{ORCID}

Ung Kim, https://orcid.org/0000-0002-6009-1843

\section{REFERENCES}

1. Cribier A, Eltchaninoff H, Bash A, Borenstein N, Tron C, Bauer F, et al. Percutaneous transcatheter implantation of an aortic valve prosthesis for calcific aortic stenosis: first human case description. Circulation 2002;106:3006-8.

2. Nishimura RA, Otto CM, Bonow RO, Carabello BA, Erwin JP 3rd, Fleisher LA, et al. 2017 AHA/ACC focused update of the 2014 AHA/ACC guideline for the management of patients with valvular heart disease: a report of the American College of Cardiology/American Heart Association Task Force on Clinical Practice Guidelines. Circulation 2017;135: e1159-95. 
3. Vahl TP, Kodali SK, Leon MB. Transcatheter aortic valve replacement 2016: a modern-day "Through the LookingGlass” adventure. J Am Coll Cardiol 2016;67:1472-87.

4. Iung B, Cachier A, Baron G, Messika-Zeitoun D, Delahaye F, Tornos P, et al. Decision-making in elderly patients with severe aortic stenosis: why are so many denied surgery? Eur Heart J 2005;26:2714-20.

5. Krasopoulos G, Falconieri F, Benedetto U, Newton J, Sayeed $\mathrm{R}$, Kharbanda R, et al. European real world trans-catheter aortic valve implantation: systematic review and meta-analysis of European national registries. J Cardiothorac Surg 2016; 11:159.

6. Baumgartner H, Falk V, Bax JJ, De Bonis M, Hamm C, Holm PJ, et al. 2017 ESC/EACTS Guidelines for the management of valvular heart disease. Eur Heart J 2017;38:2739-91.

7. Mylotte D, Lefevre T, Søndergaard L, Watanabe Y, Modine T, Dvir D, et al. Transcatheter aortic valve replacement in bicuspid aortic valve disease. J Am Coll Cardiol 2014;64: 2330-9.

8. Yoon SH, Sharma R, Chakravarty T, Kawamori H, Maeno $\mathrm{Y}$, Miyasaka M, et al. Clinical outcomes and prognostic factors of transcatheter aortic valve implantation in bicuspid aortic valve patients. Ann Cardiothorac Surg 2017;6:463-72.

9. Dvir D, Webb JG, Bleiziffer S, Pasic M, Waksman R, Kodali $\mathrm{S}$, et al. Transcatheter aortic valve implantation in failed bioprosthetic surgical valves. JAMA 2014;312:162-70.

10. Webb JG, Mack MJ, White JM, Dvir D, Blanke P, Herrmann HC, et al. Transcatheter aortic valve implantation within degenerated aortic surgical bioprostheses: PARTNER 2 valve-in-valve registry. J Am Coll Cardiol 2017;69:2253-62.

11. Deeb GM, Chetcuti SJ, Reardon MJ, Patel HJ, Grossman PM, Schreiber T, et al. 1-year results in patients undergoing transcatheter aortic valve replacement with failed surgical bioprostheses. JACC Cardiovasc Interv 2017;10:1034-44.

12. Barbanti M, Webb JG, Gilard M, Capodanno D, Tamburino C. Transcatheter aortic valve implantation in 2017: state of the art. EuroIntervention 2017;13(AA):AA11-21.

13. Allen KB, Chhatriwalla AK, Cohen DJ, Saxon JT, Aggarwal $\mathrm{S}$, Hart A, et al. Bioprosthetic valve fracture to facilitate transcatheter valve-in-valve implantation. Ann Thorac Surg 2017; 104:1501-8.

14. Franzone A, Piccolo R, Siontis GC, Lanz J, Stortecky S, Praz F, et al. Transcatheter aortic valve replacement for the treatment of pure native aortic valve regurgitation: a systematic review. JACC Cardiovasc Interv 2016;9:2308-17.

15. Sawaya FJ, Deutsch MA, Seiffert M, Yoon SH, Codner P, Wickramarachchi U, et al. Safety and efficacy of transcatheter aortic valve replacement in the treatment of pure aortic regurgitation in native valves and failing surgical bioprostheses: results from an international registry study. JACC Cardiovasc Interv 2017;10:1048-56.

16. Wei L, Liu H, Zhu L, Yang Y, Zheng J, Guo K, et al. A new transcatheter aortic valve replacement system for predominant aortic regurgitation implantation of the J-valve and early outcome. JACC Cardiovasc Interv 2015;8:1831-41.

17. Thyregod HG, Steinbrüchel DA, Ihlemann N, Nissen H,
Kjeldsen BJ, Petursson P, et al. Transcatheter versus surgical aortic valve replacement in patients with severe aortic valve stenosis: 1-year results from the all-comers NOTION randomized clinical trial. J Am Coll Cardiol 2015;65:2184-94.

18. Leon MB, Smith CR, Mack MJ, Makkar RR, Svensson LG, Kodali SK, et al. Transcatheter or surgical aortic-valve replacement in intermediate-risk patients. N Engl J Med 2016; 374:1609-20.

19. Reardon MJ, Van Mieghem NM, Popma JJ, Kleiman NS, Søndergaard L, Mumtaz M, et al. Surgical or transcatheter aortic-valve replacement in intermediate-risk patients. N Engl J Med 2017;376:1321-31.

20. Barbanti M, Binder RK, Freeman M, Wood DA, Leipsic J, Cheung A, et al. Impact of low-profile sheaths on vascular complications during transfemoral transcatheter aortic valve replacement. EuroIntervention 2013;9:929-35.

21. Barbanti M, Buccheri S, Rodés-Cabau J, Gulino S, Généreux $P$, Pilato G, et al. Transcatheter aortic valve replacement with new-generation devices: a systematic review and meta-analysis. Int J Cardiol 2017;245:83-9.

22. Pascual I, Carro A, Avanzas P, Hernández-Vaquero D, Díaz R, Rozado J, et al. Vascular approaches for transcatheter aortic valve implantation. J Thorac Dis 2017;9(Suppl 6): S47887.

23. Arsalan M, Walther T. Durability of prostheses for transcatheter aortic valve implantation. Nat Rev Cardiol 2016;13: 360-7.

24. Latib A, Naganuma T, Abdel-Wahab M, Danenberg H, Cota L, Barbanti M, et al. Treatment and clinical outcomes of transcatheter heart valve thrombosis. Circ Cardiovasc Interv 2015; 8. pii: e001779.

25. Chakravarty T, Søndergaard L, Friedman J, De Backer O, Berman D, Kofoed KF, et al. Subclinical leaflet thrombosis in surgical and transcatheter bioprosthetic aortic valves: an observational study. Lancet 2017;389:2383-92.

26. Toggweiler S, Leipsic J, Binder RK, Freeman M, Barbanti $\mathrm{M}$, Heijmen $\mathrm{RH}$, et al. Management of vascular access in transcatheter aortic valve replacement: part 2: vascular complications. JACC Cardiovasc Interv 2013;6:767-76.

27. Auffret V, Regueiro A, Del Trigo M, Abdul-Jawad Altisent O, Campelo-Parada F, Chiche O, et al. Predictors of early cerebrovascular events in patients with aortic stenosis undergoing transcatheter aortic valve replacement. J Am Coll Cardiol 2016;68:673-84.

28. Kahlert P, Al-Rashid F, Döttger P, Mori K, Plicht B, Wendt $\mathrm{D}$, et al. Cerebral embolization during transcatheter aortic valve implantation: a transcranial Doppler study. Circulation 2012;126:1245-55.

29. Giustino G, Sorrentino S, Mehran R, Faggioni M, Dangas G. Cerebral embolic protection during TAVR: a clinical event meta-analysis. J Am Coll Cardiol 2017;69:465-6.

30. Barbanti M, Gulino S, Costa G, Tamburino C. Pathophysiology, incidence and predictors of conduction disturbances during Transcatheter Aortic Valve Implantation. Expert Rev Med Devices 2017;14:135-47.

31. Généreux P, Head SJ, Van Mieghem NM, Kodali S, Kirtane 
$\mathrm{AJ}, \mathrm{Xu} \mathrm{K}$, et al. Clinical outcomes after transcatheter aortic valve replacement using valve academic research consortium definitions: a weighted meta-analysis of 3,519 patients from 16 studies. J Am Coll Cardiol 2012;59:2317-26.

32. Athappan G, Gajulapalli RD, Sengodan P, Bhardwaj A, Ellis SG, Svensson L, et al. Influence of transcatheter aortic valve replacement strategy and valve design on stroke after transcatheter aortic valve replacement: a meta-analysis and systematic review of literature. J Am Coll Cardiol 2014;63: 2101-10.

33. Barbanti $M$, Sgroi $C$, Immè $S$, Aruta $P$, Deste $W$, Gulino $S$, et al. Usefulness of contrast injection during balloon aortic valvuloplasty before transcatheter aortic valve replacement: a pilot study. EuroIntervention 2014;10:241-7.

34. Regueiro A, Linke A, Latib A, Ihlemann N, Urena M, Walther $\mathrm{T}$, et al. Association between transcatheter aortic valve replacement and subsequent infective endocarditis and in-hospital death. JAMA 2016;316:1083-92.

35. Barbanti M, Webb JG, Tamburino C, Van Mieghem NM,
Makkar RR, Piazza N, et al. Outcomes of redo transcatheter aortic valve replacement for the treatment of postprocedural and late occurrence of paravalvular regurgitation and transcatheter valve failure. Circ Cardiovasc Interv 2016;9. pii: e003930.

36. Babaliaros V, Devireddy C, Lerakis S, Leonardi R, Iturra SA, Mavromatis $\mathrm{K}$, et al. Comparison of transfemoral transcatheter aortic valve replacement performed in the catheterization laboratory (minimalist approach) versus hybrid operating room (standard approach): outcomes and cost analysis. JACC Cardiovasc Interv 2014;7:898-904.

37. Barbanti M, Capranzano P, Ohno Y, Attizzani GF, Gulino $\mathrm{S}$, Immè $\mathrm{S}$, et al. Early discharge after transfemoral transcatheter aortic valve implantation. Heart 2015;101:1485-90.

38. Barbanti M, Todaro D, Costa G, Pilato G, Picci A, Gulino $\mathrm{S}$, et al. Optimized screening of coronary artery disease with invasive coronary angiography and ad hoc percutaneous coronary intervention during transcatheter aortic valve replacement. Circ Cardiovasc Interv 2017;10. pii: e005234. 